\title{
Concept of intelligent supporting information system for development of new appliances
}

\author{
Ogrizek, B. ${ }^{\text {a, }}{ }^{*}$, Rehar, T. ${ }^{b}$, Leber, M. ${ }^{c}$, Buchmeister, B. $^{\mathrm{C}}$ \\ ${ }^{a}$ BSH Home appliances, Nazarje, Slovenia \\ ${ }^{b}$ Gorenje d.d., Velenje, Slovenia \\ 'University of Maribor, Faculty of Mechanical Engineering, Maribor, Slovenia
}

\begin{abstract}
A B S T R A C T
There is increasing momentum in industrial practice to improve the development process itself. One of the key factors to do so is the desire for the profit made per appliance. The second reason is increase of the development oriented companies and with this also fierce competition in the global market. Development of the appliance lasts from the first idea about the product till the end of production. In between that time a lot of activities take place in order to achieve the success on the market. But because, as we know from the other fields, of more competition, the terms for success get stricter. This means that equally progressing companies can't make a significant competitive advantage on the market. If there is too much resources applied in the development phase it later shows on the price of the product itself. If there is too less resources assigned to it could happen the product is qualitatively not sufficient and therefore market rejects it. Therefore optimum development would be to minimize resources to the minimum necessary quantity with preserving quality of the end product. In this article there is suggested a method for helping the appliance developers, which based on existing knowledge foresees potential solutions with new challenges. To implement the system there were data collected from various beverage appliances. The main methods within the system were neural networks so the acquired data were set as a base for creating supporting neural networks. System was tested with the data that were previously not included into learning patterns. Results show the method to be suitable during new appliance development. System itself predicted expected solutions well enough to confirm usability for development purposes.
\end{abstract}

\author{
ARTICLE INFO \\ Keywords: \\ Product development \\ Home appliances \\ Neural networks \\ Intelligent system \\ Supporting information \\ *Corresponding author: \\ bostjan.ogrizek@bshg.com \\ (Ogrizek, B.)
}

Article history:

Received 3 February 2017

Revised 28 April 2017

Accepted 9 May 2017

\section{References}

[1] Brglez, Š., Dolšak, B. (2016). A necessity-based method for product requirement elicitation and classification, Transactions of FAMENA, Vol. 40, No. 1, 87-110.

[2] Koren, R., Palčič, I. (2015). The impact of technical and organisational innovation concepts on product characteristics, Advances in Production Engineering \& Management, Vol. 10, No. 1, 27-39, doi: 10.14743/apem2015.1.190.

[3] Koren, R., Prester, J., Buchmeister, B., Palčič, I. (2016). Do organisational innovations have impact on launching new products on the market?, Strojniški vestnik - Journal of Mechanical Engineering, Vol. 62, No. 6, 389-397, doi: 10.5545/sv-jme.2016.3470.

[4] Karakašić, M., Zadnik, Z., Kljajin, M., Duhovnik, J. (2016). Matrix of function and functionality as a tool in product development process, Tehnički vjesnik - Technical Gazette, Vol. 23, No. 5, 1295-1300, doi: 10.17559/TV$\underline{20160609095229}$. 
[5] Todic, V., Cosic, I., Maksimovic, R., Tasic, N., Radakovic, N. (2017). Model for simulation of life cycle costs at the stage of product development, International Journal of Simulation Modelling, Vol. 16, No. 1, 108-120, doi: 10.2507/IJSIMM16(1)9.373.

[6] Peško, I., Trivunić, M., Cirović, G., Mučenski, V. (2013). A preliminary estimate of time and cost in urban road construction using neural networks, Tehnički vjesnik - Technical Gazette, Vol. 20, No. 3, 563-570.

[7] Vahabli, E., Rahmati, S. (2016). Application of an RBF neural network for FDM parts' surface roughness prediction for enhancing surface quality, International Journal of Precision Engineering and Manufacturing, Vol. 17, No. 12, 1589-1603, doi: $10.1007 / \mathrm{s} 12541-016-0185-7$.

[8] Saric, T., Simunovic, G., Simunovic, K. (2013). Use of neural networks in prediction and simulation of steel surface roughness, International Journal of Simulation Modelling, Vol. 12, No. 4, 225-236, doi: 10.2507/IJSIMM12 (4) 2.241.

[9] Vijayaraghavan, V., Garg, A., Lam, J.S.L., Panda, B., Mahapatra, S.S. (2015). Process characterisation of 3D-printed FDM components using improved evolutionary computational approach, The International Journal of Advanced Manufacturing Technology, Vol. 78, No. 5, 781-793, doi: 10.1007/s00170-014-6679-5.

[10] Paulic, M., Mocnik, D., Ficko, M., Balic, J., Irgolic, T., Klancnik, S. (2015). Intelligent system for prediction of mechanical properties of material based on metallographic images, Tehnički vjesnik - Technical Gazette, Vol. 22, No. 6, 1419-1424, doi: 10.17559/TV-20130718090927.

[11] Klancnik, S., Begic-Hajdarevic, D., Paulic, M., Ficko, M., Cekic, A., Cohodar Husic, M. (2015). Prediction of laser cut quality for Tungsten alloy using the neural network method, Strojniski vestnik - Journal of Mechanical Engineering, Vol. 61, No. 12, 714-720, doi: 10.5545/sv-jme.2015.2717.

[12] Garg, A., Tai, K., Lee, C.H., Savalani, M.M. (2014). A hybrid M5'-genetic programming approach for ensuring greater trustworthiness of prediction ability in modelling of FDM process, Journal of Intelligent Manufacturing, Vol. 25, No. 6, 1349-1365, doi: 10.1007/s10845-013-0734-1.

[13] Wuest, T., Weimer, D., Irgens, C., Thoben, K.-D. (2016). Machine learning in manufacturing: Advantages, challenges, and applications, Production \& Manufacturing Research, Vol. 4, No. 1, 23-45, doi: 10.1080/21693277. 2016.1192517.

[14] Wang, G., Tian, X., Geng, J., Guo, B. (2015). A knowledge accumulation approach based on bilayer social wiki network for computer-aided process innovation, International Journal of Production Research, Vol. 53, No. 8, 2365-2382, doi: 10.1080/00207543.2014.958591.

[15] Comesaña-Campos, A., Bouza-Rodríguez, J.B. (2016). An application of Hebbian learning in the design process decision-making, Journal of Intelligent Manufacturing, Vol. 27, No. 3, 487-506, doi: 10.1007/s10845-014-0881-z.

[16] Wu, H., Evans, G., Bae, K.-H. (2016). Production control in a complex production system using approximate dynamic programming, International Journal of Production Research, Vol. 54, No. 8, 2419-2432, doi: $10.1080 / 00207543.2015 .1086035$.

[17] Xu, Y., Chen, G., Zheng, J. (2016). An integrated solution - KAGFM for mass customization in customer-oriented product design under cloud manufacturing environment, The International Journal of Advanced Manufacturing Technology, Vol. 84, No. 1, 85-101, doi: 10.1007/s00170-015-8074-2.

[18] Liu, J., Yu, G., Li, Y., Wang, H., Xiao, W. (2016). Multidisciplinary design optimization of crankshaft structure based on cooptimization and multi-island genetic algorithm, Mathematical Problems in Engineering, 1-11, doi: $10.1155 / 2016 / 9596089$.

[19] Kwong, C.K., Jiang, H., Luo, X.G. (2016). AI-based methodology of integrating affective design, engineering, and marketing for defining design specifications of new products, Engineering Applications of Artificial Intelligence, Vol. 47, 49-60, doi: 10.1016/i.engappai.2015.04.001.

[20] Noorossana, R., Zadbood, A., Zandi, F., Noghondarian, K. (2015). An interactive artificial neural networks approach to multiresponse optimization, The International Journal of Advanced Manufacturing Technology, Vol. 76, No. 5, 765-777, doi: 10.1007/s00170-014-6276-7.

[21] Šibalija, T., Majstorović, V., Soković, M. (2011). Taguchi-based and intelligent optimisation of a multi-response process using historical data, Strojniški vestnik - Journal of Mechanical Engineering, Vol. 57, No. 4, 357-365, doi: $10.5545 /$ sv-jme.2010.061.

[22] Lin, M.-C., Lin, Y.-H., Lin, C.-C., Chen, M.-S., Hung, Y.-C. (2015). An integrated neuro-genetic approach incorporating the Taguchi method for product design, Advanced Engineering Informatics, Vol. 29, No. 1, 47-58, doi: 10.1016/j.aei.2014.09.002.

[23] Duan, W., An, L., Wang, Z. (2014). Strength reliability analysis of turbine blade using surrogate models, Research Journal of Applied Sciences, Engineering \& Technology, Vol. 7, No. 18, 3699-3708, doi: 10.19026/rjaset.7.724.

[24] Chen, H.-Y., Chang, Y.-M. (2014). Development of a computer aided product-form design tool based on numerical definition scheme and neural network, Journal of Advanced Mechanical Design, Systems, and Manufacturing, Vol. 8, No. 3, 1-15, doi: 10.1299/jamdsm.2014jamdsm0033.

[25] Tang, C.Y., Fung, K.Y., Lee, E.W.M., Ho, G.T.S., Siu, K.W.M., Mou, W.L. (2013). Product form design using customer perception evaluation by a combined superellipse fitting and ANN approach, Advanced Engineering Informatics, Vol. 27, No. 3, 386-394, doi: 10.1016/i.aei.2013.03.006.

[26] Chen, H.Q., Honda, T., Yang, M.C. (2013). Approaches for identifying consumer preferences for the design of technology products: A case study of residential solar panels, Journal of Mechanical Design, Vol. 135, No. 6, 1-12, doi: $10.1115 / 1.4024232$.

[27] Žilková, J., Timko, J., Girovský, P. (2012). Modelling and control of tinning line entry section using neural networks, International Journal of Simulation Modelling, Vol. 11, No. 2, 97-109, doi: 10.2507/IJSIMM11(2)4.210.

[28] Edwin Raja Dhas, J., Kumanan, S. (2013). Modeling and prediction of HAZ using finite element and neural network modeling, Advances in Production Engineering \& Management, Vol. 8, No. 1, 13-24, doi: 10.14743/ apem2013.1.149. 
[29] Kutschenreiter-Praszkiewicz, I. (2013). Application of neural network in QFD matrix, Journal of Intelligent Manufacturing, Vol. 24, No. 2, 397-404, doi: 10.1007/s10845-011-0604-7.

[30] Taha, B.O., Ali, P.J.M., Ahmed, H.A. (2015). Optimizing the flexural strength of beams reinforced with fiber reinforced polymer bars using back-propagation neural networks, ARO The Scientific Journal of Koya University, Vol. 3, No. 2, 1-10, doi: 10.14500/aro.10066.

[31] Kiliç, N., Ekici, B., Hartomacıoğlu, S. (2015). Determination of penetration depth at high velocity impact using finite element method and artificial neural network tools, Defence Technology, Vol. 11, No. 2, 110-122, doi: 10.1016/j.dt.2014.12.001. 


\section{APEM}

Advances in Production Engineering \& Management

Letnik 12 | Številka 2 | Junij 2017 | Strani 196-204

https://doi.org/10.14743/apem2017.2.251
ISSN 1854-6250

Spletna stran: apem-journal.org Izvirni znanstveni članek

\title{
Zasnova inteligentnega podpornega informacijskega sistema za razvoj novih gospodinjskih aparatov
}

\author{
Ogrizek, B. ${ }^{a,}{ }^{*}$, Rehar, T. ${ }^{b}$, Leber, M. ${ }^{c}$, Buchmeister, B. ${ }^{c}$ \\ ${ }^{a}$ BSH Home appliances, Nazarje, Slovenia \\ ${ }^{b}$ Gorenje d.d., Velenje, Slovenia \\ 'University of Maribor, Faculty of Mechanical Engineering, Maribor, Slovenia
}

\begin{abstract}
POVZETEK
V industrijski praksi je opazna težnja po izboljšanju razvojnega procesa. Eden od ključnih dejavnikov za to je želja po dobičku na izdelek. Drugi razlog je povečanje števila razvojno usmerjenih podjetij in ostre konkurence na svetovnem trgu. Razvoj aparatov traja od prve zamisli o izdelku do zaključka proizvodnje. V tem času potekajo številne dejavnosti, da bi dosegli uspeh na trgu. Kot opaža-mo vse večjo konkurenčnost na vseh področjih, postajajo tudi pogoji za uspeh vse hujši. To pomeni, da enako napredujoča podjetja ne morejo imeti pomembnejše konkurenčne prednosti na trgu. Če se v razvojni fazi uporablja preveč sredstev, se kasneje to pokaže na ceni samega izdelka. Če je dodeljenih premalo sredstev, se lahko zgodi, da bo izdelek kakovostno nezadosten in ga bo trg zavrnil. Zato bi bil optimalen razvoj z minimizacijo virov do najmanjše potrebne količine ob ohranjanju kakovosti končnega izdelka. V tem članku je predlagana metoda za pomoč razvijalcem gospodinjskih aparatov, ki na podlagi obstoječega znanja predvideva možne rešitve z novimi izzivi. Za izvedbo sistema so bili zbrani podatki o različnih aparatih za pripravo napitkov. Glavna uporabljena metoda v sistemu so bile nevronske mreže, tako da so pridobljeni podatki služili kot osnova za ustvarjanje podpornih nevronskih mrež. Sistem je bil preskušen s podatki, ki niso bili vključeni v učne vzorce. Rezultati kažejo, da je metoda primerna za potrebe razvoja novih aparatov. Sistem sam napoveduje pričakovane rešitve, ki so dovolj zanesljive, da lahko potrdimo njegovo uporabnosti za razvojne namene.
\end{abstract}

PODATKI O ČLANKU

Ključne besede:

Razvoj izdelkov

Gospodinjski aparati

Nevronske mreže

Inteligentni sistem

Podporne informacije

*Kontaktna oseba:

bostjan.ogrizek@bshg.com

(Ogrizek, B.)

Zgodovina članka:

Prejet 3. februarja 2017

Popravljen 28. aprila 2017

Sprejet 9. maja 2017 